\title{
Uncertain systems are universal approximators
}

\author{
Zixiong Peng ${ }^{1}$ and Xiaowei Chen ${ }^{2 *}$
}

\author{
${ }^{*}$ Correspondence: \\ chenx@nankai.edu.cn \\ ${ }^{2}$ Department of Risk Management \\ and Insurance, Nankai University, \\ Tianjin 300071, China \\ Full list of author information is \\ available at the end of the article
}

\begin{abstract}
Uncertain inference is a process of deriving consequences from uncertain knowledge or evidences via the tool of conditional uncertain set. Based on uncertain inference, uncertain system is a function from its inputs to outputs. This paper proves that uncertain systems are universal approximators, which means that uncertain systems are capable of approximating any continuous function on a compact set to arbitrary accuracy. This result can be viewed as an existence theorem of an optimal uncertain system for a wide variety of problems.
\end{abstract}

Keywords: Uncertainty theory; Uncertain inference; Uncertain system; Universal approximation

\section{Introduction}

Fuzzy systems were developed from fuzzy set theory initialized by Zadeh [1] in 1965. In recent decades, fuzzy systems have been successfully applied to a wide variety of practical problems, such as fuzzy control, fuzzy identification, fuzzy expert system. Generally speaking, there are two types of widely used fuzzy systems: Mamdani fuzzy systems [2] and Takagi-Sugeno fuzzy systems [3]. The main difference between these fuzzy systems lies on inference rules, such as Zadeh's compositional rule of inference, Lukasiewicz's inference rule [4], Mamdani's inference rules [2,5] for Mamdani fuzzy systems. On the other hand, Wang [6] proved that fuzzy systems are universal approximators which is an important theoretical basis for the application of it.

Although fuzzy systems are successfully used in engineering problems, it isn't perfect in its theoretical framework. For example, membership functions are adopted to describe the fuzzy sets used in fuzzy inference rules, however, the overuse maximum operations in fuzzy set theory is impeached. Surveys have shown that the inputs and outputs of practical systems should not be fuzzy sets. This promotes Liu [7] to define uncertain set and introduced uncertain systems based Liu's inference rules. Then, Gao Gao and Ralescu [8] extended the Liu's inference rule to multi-antecedent. As an application of uncertain system, Gao [9] used the uncertain controller to successfully control the inverted pendulum. Besides, uncertain logic was introduced by Liu [10] via uncertain set. Universal approximation capability of uncertain systems is the basis of almost all the theoretical research and practical applications of uncertain systems. In this paper, we will prove that uncertain systems are universal approximators, and this result can be viewed as an existence theorem of an optimal uncertain system for a wide variety of problems. 
The rest of the paper is organized as follows. Section 'Preliminaries' introduces some basic concepts and results on uncertain sets as preliminaries. The framework of Liu's inference rule and uncertain systems are introduced in Section 'Inference rule and uncertain systems'. It is shown that uncertain systems are universal approximators in Section 'Uncertain systems are universal approximators'. Section 'Conclusions' concludes this paper with a brief summary.

\section{Preliminaries}

Liu [11] founded an uncertainty theory that is based on normality, duality, subadditivity and product axioms. Gao [12] studied some properties of uncertain measure. Since then, considerable work has been done based on uncertainty theory. Liu [13] founded uncertain programming to model optimization problems in uncertain environments. Liu [14] proposed the concept of uncertain process and uncertain calculus. Li and Liu [15] proposed uncertain logic. And Liu [16] studied entailment based on uncertain proposition logic. Recently, uncertain systems are suggested by Liu [7] in 2010 via uncertain inference and uncertain set theory. Uncertainty theory has become a new tool to describe belief degree and has applications in practical use. For a detailed exposition, the interested reader may consult the book [17] to know more about uncertainty theory. In this section, we will introduce some definitions related on uncertain systems. Let $\Gamma$ be a nonempty set, and let $\mathrm{L}$ be a $\sigma$-algebra over $\Gamma$. Each element $\Lambda \in \Gamma$ is called an event. In order to measure uncertain events, Liu [11] introduced an uncertain measure $\mathcal{M}$ as a set function satisfying normality, self-duality, and countable subadditivity axioms. Then the triplet $(\Gamma, \mathrm{L}, \mathcal{M})$ is called an uncertainty space.

Let $\Gamma$ be a nonempty set, and $\mathcal{L}$ a $\sigma$-algebra over $\Gamma$. An uncertain measure $\mathcal{M}$ (Liu [11]) is a set function defined on $\mathcal{L}$ satisfying the following four axioms:

Axiom 1. (Normality Axiom) $\mathcal{M}\{\Gamma\}=1$;

Axiom 2. (Duality Axiom) $\mathcal{M}\{\Lambda\}+\mathcal{M}\left\{\Lambda^{c}\right\}=1$ for any event $\Lambda$;

Axiom 3. (Subadditivity Axiom) For every countable sequence of events $\left\{\Lambda_{i}\right\}$, we have

$$
\mathcal{M}\left\{\bigcup_{i=1}^{\infty} \Lambda_{i}\right\} \leq \sum_{i=1}^{\infty} \mathcal{M}\left\{\Lambda_{i}\right\} .
$$

Axiom 4. (Product Axiom [18]) Let $\left(\Gamma_{k}, \mathrm{~L}_{k}, \mathcal{M}_{k}\right)$ be uncertainty spaces for $k=1,2, \ldots$. The product uncertain measure $\mathcal{M}$ is an uncertain measure satisfying

$$
\mathcal{M}\left\{\prod_{i=1}^{\infty} \Lambda_{i}\right\}=\bigwedge_{i=1}^{\infty} \mathcal{M}\left\{\Lambda_{i}\right\}
$$

where $\Lambda_{k}$ are arbitrarily chosen events from $L_{k}$ for $k=1,2, \ldots$, respectively.

Definition 1. (Liu [7]) An uncertain set is a measurable function $\xi$ from an uncertainty space $(\Gamma, \mathrm{L}, \mathcal{M})$ to a collection of sets, i.e., both $\{B \subset \xi\}$ and $\{\xi \subset B\}$ are events for any Borel set $B$. 
Let $\xi$ and $\eta$ be two nonempty uncertain sets. What is the appropriate event that $\xi$ is included in $\eta$ ? We may suggest that $\{\xi \subset \eta\}=\{\gamma \mid \xi(\gamma) \subset \eta\}$ intuitively. The set $\{B \not \subset$ $\xi\}=\{\gamma \mid B \not \subset \xi(\gamma)\}$ and $\{\xi \not \subset B\}=\{\gamma \mid \xi(\gamma) \not \subset B\}$ are also events.

Definition 2. (Liu [19]) An uncertain set $\xi$ is said to have a membership function $\mu$ if for any Borel set $B$ of real numbers, we have

$$
\mathcal{M}\{B \subset \xi\}=\inf _{x \in B} \mu(x), \quad \mathcal{M}\{\xi \subset B\}=1-\sup _{x \in B^{c}} \mu(x) .
$$

The above equations will be called measure inversion formulas.

Example 1. By a rectangular uncertain set we mean the uncertain set fully determined by the pair $(a, b)$ of crisp numbers with $a<b$, whose membership function is

$$
\mu(x)=1, \quad a \leq x \leq b .
$$

Example 2. By a triangular uncertain set we mean the uncertain set fully determined by the triplet $(a, b, c)$ of crisp numbers with $a<b<c$, whose membership function is

$$
\mu(x)=\left\{\begin{array}{l}
\frac{x-a}{b-a}, \text { if } a \leq x \leq b \\
\frac{x-c}{b-c}, \text { if } b \leq x \leq c .
\end{array}\right.
$$

Example 3. By a trapezoidal uncertain set we mean the uncertain set fully determined by the quadruplet $(a, b, c, d)$ of crisp numbers with $a<b<c<d$, whose membership function is

$$
\mu(x)=\left\{\begin{array}{c}
\frac{x-a}{b-a}, \text { if } a \leq x \leq b \\
1, \quad \text { if } b \leq x \leq c \\
\frac{x-d}{c-d}, \text { if } c \leq x \leq d .
\end{array}\right.
$$

Liu [17] proved that a real-valued function $\mu$ is a membership function if and only if $0 \leq \mu(x) \leq 1$.

Definition 3. (Liu [19]) Let $\xi$ be an uncertain set with membership function $\mu$. Then the set-valued function

$$
\mu^{-1}(\alpha)=\{x \in \Re \mid \mu(x) \geq \alpha\}
$$

is called the inverse membership function of $\xi$. Sometimes, the set $\mu^{-1}(\alpha)$ is called the $\alpha$-cut of $\mu$.

The rectangular uncertain set $\xi=(a, b)$ has an inverse membership function

$$
\mu^{-1}(\alpha) \equiv[a, b] .
$$

The triangular uncertain set $\xi=(a, b, c)$ has an inverse membership function

$$
\mu^{-1}(\alpha)=[(1-\alpha) a+\alpha b, \alpha b+(1-\alpha) c] .
$$


The trapezoidal uncertain set $\xi=(a, b, c, d)$ has an inverse membership function

$$
\mu^{-1}(\alpha)=[(1-\alpha) a+\alpha b, \alpha c+(1-\alpha) d] .
$$

Liu [17] proposed the conditional membership function of an uncertain set $\xi$ after some event $A$ has occurred:

$$
\mathcal{M}\{B \subset \xi \mid A\}=\left\{\begin{array}{cl}
\frac{\mathcal{M}\{(B \subset \xi) \cap(\xi \subset A)\}}{\mathcal{M}\{\xi \subset A\}}, & \text { if } \frac{\mathcal{M}\{(B \subset \xi) \cap(\xi \subset A)\}}{\mathcal{M}\{\xi \subset A\}}<0.5 \\
1-\frac{\mathcal{M}\{(B \not \subset \xi) \cap(\xi \subset A)\}}{\mathcal{N}\{\xi \subset A\}}, & \text { if } \frac{\mathcal{M}\{(B \not \subset \xi) \cap(\xi \subset A)\}}{\mathcal{M}\{\xi \subset A\}}<0.5 \\
0.5, & \text { otherwise }
\end{array}\right.
$$

and

$$
\mathcal{M}\{\xi \subset B \mid A\}=\left\{\begin{array}{cl}
\frac{\mathcal{M}\{(\xi \subset B) \bigcap(\xi \subset A)\}}{\mathcal{M}\{\xi \subset A\}}, & \text { if } \frac{\mathcal{M}\{(\xi \subset B) \bigcap(\xi \subset A)\}}{\mathcal{M}\{\xi \subset A\}}<0.5 \\
1-\frac{\mathcal{M}\{(\xi \not \subset B) \bigcap(\xi \subset A)\}}{\mathcal{M}\{\xi \subset A\}}, & \text { if } \frac{\mathcal{M}\{(\xi \not \subset B) \bigcap(\xi \subset A)\}}{\mathcal{M}\{\xi \subset A\}}<0.5 \\
0.5, & \text { otherwise. }
\end{array}\right.
$$

Definition 4. (Liu [20]) The uncertain sets $\xi_{1}, \xi_{2}, \ldots, \xi_{n}$ are said to be independent if for any Borel sets $B_{1}, B_{2}, \ldots, B_{n}$, we have

$$
\mathcal{M}\left\{\bigcap_{i=1}^{n}\left(\xi_{i}^{*} \subset B_{i}\right)\right\}=\bigwedge_{i=1}^{n} \mathcal{M}\left\{\xi_{i}^{*} \subset B_{i}\right\}
$$

and

$$
\mathcal{M}\left\{\bigcup_{i=1}^{n}\left(\xi_{i}^{*} \subset B_{i}\right)\right\}=\bigvee_{i=1}^{n} \mathcal{M}\left\{\xi_{i}^{*} \subset B_{i}\right\}
$$

where $\xi_{i}^{*}$ are arbitrarily chosen from $\left\{\xi_{i}, \xi_{i}^{c}\right\}, i=1,2, \ldots, n$, respectively.

Definition 5. (Liu [20]) Let $\xi$ and $\eta$ be independent uncertain sets with membership functions $\mu$ and $\nu$, respectively. Then their union $\xi \cap \eta$ has a membership function

$$
\lambda(x)=\mu(x) \vee v(x) .
$$

Definition 6. (Liu [20]) Let $\xi$ and $\eta$ be independent uncertain sets with membership functions $\mu$ and $v$, respectively. Then their union $\xi \cup \eta$ has a membership function

$$
\lambda(x)=\mu(x) \wedge v(x) .
$$

Definition 7. (Liu [20]) Let $\xi$ be an uncertain sets with membership functions $\mu$. Then their union $\xi^{c}$ has a membership function

$$
\lambda(x)=1-v(x) .
$$

Definition 8. (Liu [20]) Let $\xi_{1}, \xi_{2}, \ldots, \xi_{n}$ be independent uncertain sets with inverse membership functions $\mu_{1}(x), \mu_{2}(x), \ldots, \mu_{n}(x)$, respectively. If $f$ is a measurable 
function, then the uncertain set $\xi=f\left(\xi_{1}, \xi_{2}, \ldots, \xi_{n}\right)$ has an inverse membership function,

$$
\mu^{-1}(\alpha)=f\left(\mu_{1}^{-1}(\alpha), \mu_{2}^{-1}(\alpha), \ldots, \mu_{n}^{-1}(\alpha)\right) .
$$

\section{Inference rule and uncertain systems}

Uncertain inference was proposed by Liu [7] as a process of deriving consequences from uncertain knowledge or evidence via the tool of conditional uncertain set. Then Gao, Gao and Ralescu [8] extended Liu's inference rule to the one with multiple antecedents and with multiple if-then rules. Besides, Gao [9] applied uncertain inference to invert pendulum. Furthermore, uncertain set was extended to the field of logic (Liu [10]) where the uncertain quantifier, uncertain subject and uncertain predicate are described by uncertain sets. In this section, we will introduce the concept and some properties of uncertain inference.

Liu's Inference Rule [7]. Let $\mathbb{X}$ and $\mathbb{Y}$ be two concepts. Assume rules if $\mathbb{X}$ is an uncertain set $\xi$ then $\mathbb{Y}$ is an uncertain set $\eta^{\prime}$. From $\mathbb{X}$ is a constant $a$, we infer that $\mathbb{Y}$ is an uncertain set

$$
\eta *=\left.\eta\right|_{a \in \eta}
$$

which is the conditional uncertain set of $\eta$ given $a \in \eta$. The inference rule is represented by

Rule: if $\mathbb{X}$ is $\xi$ then $\mathbb{Y}$ is $\eta$

From: $\mathbb{X}$ is a constant $a$

Infer: $\mathbb{Y}$ is $\eta^{*}=\left.\eta\right|_{a \in \xi}$

It has been proved by Liu [7] that the membership function of $\eta^{*}$ satisfying

$$
v^{*}(y)=\left\{\begin{array}{cl}
\frac{v(y)}{\mu(a)}, & \text { if } v(y)<\mu(a) / 2 \\
\frac{v(y)+\mu(a)-1}{\mu(a)}, & \text { if } v(y)>1-\mu(a) / 2 \\
0.5, & \text { otherwise. }
\end{array}\right.
$$

Liu's Inference Rule (multiple antecedent [8]). Let $\mathbb{X}, \mathbb{Y}$, and $\mathbb{Z}$ be three concepts. Assume rules 'if $\mathbb{X}$ is an uncertain set $\xi$ and $\mathbb{Y}$ is $\eta$ then $\mathbb{Z}$ is an uncertain set $\tau$ '. From $\mathbb{X}_{1}$ is a constant $a$ and $\mathbb{Y}$ is a constant $b$, respectively, we infer that $\mathbb{Z}$ is an uncertain set.

$$
\eta *=\left.\eta\right|_{(a \in \eta)} \cap(b \in \eta)
$$

which is conditional uncertain set of $\tau$ of given $a \in \xi$ and $b \in \eta$. The inference rule is represented by

Rule: if $\mathbb{X}$ is $\xi$ and $\mathbb{Y}$ is $\eta$ then $\mathbb{Z}$ is $\tau$

From: $\mathbb{X}$ is a constant $a$ and $\mathbb{Y}$ is a constant $b$

Infer: $\mathbb{Z}$ is $\eta^{*}=\left.\eta\right|_{(a \in \eta)} \bigcap(b \in \eta)$ 
It has been proved by Gao, Gao, and Ralescu [8] that

$$
\lambda^{*}(z)=\left\{\begin{array}{cl}
\frac{\lambda(z)}{\mu(a) \wedge v(b)}, & \text { if } \lambda(z)<\frac{\mu(a) \wedge v(b)}{2} \\
\frac{\lambda(z)+\mu(a) \wedge v(b)-1}{\mu(a) \wedge v(b)}, & \text { if } \lambda(z)>1-\frac{\mu(a) \wedge v(b)}{2} \\
0.5, & \text { otherwise. }
\end{array}\right.
$$

Liu's Inference Rule (Multiple If-Then Rules). Let $\mathbb{X}$ and $\mathbb{Y}$ be two concepts. Assume two rules 'if $\mathbb{X}$ is an uncertain set $\xi_{1}$ then $\mathbb{Y}$ is an uncertain set $\eta_{1}$ ' and 'if $\mathbb{X}$ is an uncertain set $\xi_{2}$ then $\mathbb{Y}$ is an uncertain set $\eta_{2}$ '. From $\mathbb{X}$ is a constant $a$, we infer that $\mathbb{Y}$ is an uncertain set

$$
\eta^{*}=\frac{\left.\mathcal{M}\left\{a \in \xi_{1}\right\} \cdot \eta_{1}\right|_{a \in \xi_{1}}}{\mathcal{M}\left\{a \in \xi_{1}\right\}+\mathcal{M}\left\{a \in \xi_{2}\right\}}+\frac{\left.\mathcal{M}\left\{a \in \xi_{2}\right\} \cdot \eta_{2}\right|_{a \in \xi_{2}}}{\mathcal{M}\left\{a \in \xi_{1}\right\}+\mathcal{N}\left\{a \in \xi_{2}\right\}} .
$$

The inference rule is represented by

Rule 1 : if $\mathbb{X}$ is $\xi_{1}$ then $\mathbb{Y}$ is $\eta_{1}$

Rule 2: if $\mathbb{X}$ is $\xi_{2}$ then $\mathbb{Y}$ is $\eta_{2}$

From: $\mathbb{X}$ is a constant $a$

Infer: $\mathbb{Y}$ is $\eta^{*}$

If $\xi_{1}, \xi_{2}, \eta_{1}, \eta_{2}$ are independent uncertain sets with continuous membership functions $\mu_{1}, \mu_{2}, v_{1}, v_{2}$, respectively, then the inference rule yields

$$
\eta^{*}=\frac{\mu_{1}(a)}{\mu_{1}(a)+\mu_{2}(a)} \eta_{1}^{*}+\frac{\mu_{2}(a)}{\mu_{1}(a)+\mu_{2}(a)} \eta_{2}^{*}
$$

where $\eta_{i}^{*}$ are uncertain sets whose membership functions are

$$
v_{i}^{*}(y)=\left\{\begin{array}{cl}
\frac{v_{i}(y)}{\mu_{i}(a)}, & \text { if } v_{i}(y)<\mu_{i}(a) / 2 \\
\frac{v_{i}(y)+\mu_{i}(a)-1}{\mu_{i}(a)}, & \text { if } v_{i}(y)>1-\mu_{i}(a) / 2 \\
0.5, & \text { otherwise. }
\end{array}\right.
$$

For a general system, Liu [17] proposed the following inference rule.

Liu's Inference Rule (general form) [17]. Let $\mathbb{X}_{1}, \mathbb{X}_{2}, \ldots, \mathbb{X}_{m}$ be $m$ concepts. Assume the rules if $\mathbb{X}_{1}$ is $\xi_{i 1}$ and $\cdots$ and $\mathbb{X}_{m}$ is $\xi_{i m}$, then $\mathbb{Y}$ is $\eta_{i}^{\prime}$ for $i=1,2, \ldots, k$. From $\mathbb{X}_{1}$ is $a_{1}$ and $\cdots$ and $\mathbb{X}_{m}$ is $a_{m}$, we infer that $\mathbb{Y}$ is an uncertain set

$$
\eta^{*}=\sum_{i=1}^{k} \frac{\left.c_{i} \cdot \eta_{i}\right|_{\left(a_{1} \in \xi_{i 1}\right) \cap\left(a_{2} \in \xi_{i 2}\right) \cap \cdots \cap\left(a_{m} \in \xi_{i m}\right)}}{c_{1}+c_{2}+\cdots+c_{k}}
$$

where the coefficients are determined by

$$
c_{i}=\mathcal{M}\left\{\left(a_{1} \in \xi_{i 1}\right) \cap\left(a_{2} \in \xi_{i 2}\right) \cap \cdots \cap\left(a_{m} \in \xi_{i m}\right)\right\}, i=1,2, \ldots, k .
$$

The inference rule is represented by

Rule 1: If $\mathbb{X}_{1}$ is $\xi_{11}$ and $\cdots$ and $\mathbb{X}_{m}$ is $\xi_{1 m}$ then $\mathbb{Y}$ is $\eta_{1}$

Rule 2: If $\mathbb{X}_{1}$ is $\xi_{21}$ and $\cdots$ and $\mathbb{X}_{m}$ is $\xi_{2 m}$ then $\mathbb{Y}$ is $\eta_{2}$

Rule $k$ : If $\mathbb{X}_{1}$ is $\xi_{k 1}$ and $\cdots$ and $\mathbb{X}_{m}$ is $\xi_{k m}$ then $\mathbb{Y}$ is $\eta_{k}$

From: $\mathbb{X}_{1}$ is $a_{1}$ and $\cdots$ and $\mathbb{X}_{m}$ is $a_{m}$

Infer: $\mathbb{Y}$ is $\eta^{*}$ 
Assume $\xi_{i 1}, \xi_{i 2}, \ldots, \xi_{i m}, \eta_{i}$ are independent uncertain sets with membership functions $\mu_{i 1}, \mu_{i 2}, \ldots, \mu_{i m}, v_{i}, i=1,2, \ldots, k$, respectively. If $\xi_{1}^{*}, \xi_{2}^{*}, \ldots, \xi_{m}^{*}$ are constants $a_{1}, a_{2}, \ldots, a_{m}$, respectively. It has been proved by Liu [17] that

$$
\eta^{*}=\sum_{i=1}^{k} \frac{c_{i} \cdot \eta_{i}^{*}}{c_{1}+c_{2}+\cdots+c_{k}}
$$

where $\eta_{i}^{*}$ are uncertain sets whose membership functions are given by

$$
v_{i}^{*}(y)=\left\{\begin{array}{cl}
\frac{v_{i}(y)}{c_{i}}, & \text { if } v_{i}(y)<c_{i} / 2 \\
\frac{v_{i}(y)+c_{i}-1}{c_{i}}, & \text { if } v_{i}(y)>1-c_{i} / 2 \\
0.5, & \text { otherwise. }
\end{array} \quad c_{i}=\min _{1 \leq l \leq m} \mu_{i l}\left(a_{l}\right)\right.
$$

\section{Uncertain systems are universal approximators}

An uncertain system, introduced by Liu [7], is a function from its inputs to outputs via Liu's inference rule, in which five parts are contained: input, a rule-base, Liu's inference rule, an expected value operator and outputs. An uncertain system is a function $f:\left(\alpha_{1}, \alpha_{2}, \cdots, \alpha_{m}\right) \rightarrow\left(\beta_{1}, \beta_{2}, \cdots, \beta_{n}\right)$, namely,

$$
\left(\beta_{1}, \beta_{2}, \cdots, \beta_{n}\right)=f\left(\alpha_{1}, \alpha_{2}, \cdots, \alpha_{m}\right) .
$$

Then we get an uncertain system $f$. Gao [9] has succeeded in controlling inverted pendulum. In this section, we will prove that uncertain systems are universal approximators. That is, uncertain systems are capable of approximating any continuous function on a compact set to arbitrary accuracy. Since there are great flexibilities in constructing rules, we have different methods to construct uncertain systems. The quantity of Liu's inference rules in an uncertain system is under consideration.

Theorem 1. For any given continuous function $g$ on a compact set $D \subset \Re^{m}$, and any given $\epsilon>0$, there exists an uncertain system $f$ such that

$$
\sup _{\left(\alpha_{1}, \alpha_{2}, \ldots, \alpha_{m}\right) \in D}|| f\left(\alpha_{1}, \alpha_{2}, \ldots, \alpha_{m}\right)-g\left(\alpha_{1}, \alpha_{2}, \ldots, \alpha_{m}\right) \|<\epsilon
$$

Proof. Without loss of generality, assume $g$ is a real-valued function with two variables $\alpha_{1}$ and $\alpha_{2}$ and $D=[0,1]^{2}$. Since $D$ is a compact set and $g$ is a continuous function, we know that $g$ is uniformly continuous on $D$. Thus, for any given number $\epsilon>0$, there exists a number $\delta>0$ such that

$$
\mid g\left(\alpha_{1}, \alpha_{2}\right)-g\left(\alpha_{1}^{\prime}, \alpha_{2}^{\prime}\right)<\epsilon,\left(\alpha_{1}, \alpha_{2}\right),\left(\alpha_{1}^{\prime}, \alpha_{2}^{\prime}\right) \in D
$$

where $\left\|\left(\alpha_{1}, \alpha_{2}\right)-\left(\alpha_{1}^{\prime}, \alpha_{2}^{\prime}\right)\right\|<\delta$. Let $k$ be the minimum integer larger than $1 /(\sqrt{2} \delta)$. Let

$$
D_{i, j}=\left\{\left(\alpha_{1}, \alpha_{2}\right) \mid \frac{i-1}{k}<\alpha_{1} \leq \frac{i}{k}, \frac{j-1}{k}<\alpha_{2} \leq \frac{j}{k}\right\}, i, j=1,2, \ldots, k .
$$


Note that $D_{i, j}$ are disjoint sets and $\left\|\left(\alpha_{1}, \alpha_{2}\right)-(i / k, j / k)\right\| \leq \delta$, wherever $\left(\alpha_{1}, \alpha_{2}\right) \in D_{i, j}$. Let $\xi_{i}$ and $\eta_{j}$ be independent rectangular uncertain sets with membership functions $\mu_{i}$ and $v_{i}$

$$
\mu_{i}(x)=\left\{\begin{array}{ll}
1, & \text { if } \frac{i-1}{k}<x \leq \frac{i}{k} \\
0, & \text { otherwise }
\end{array} \text { and } v_{i}(x)= \begin{cases}1, & \text { if } \frac{j-1}{k}<x \leq \frac{j}{k} \\
0, & \text { otherwise }\end{cases}\right.
$$

where $i, j=1,2, \ldots, k$, respectively. Next, we construct a rule-base like,

$$
\text { If } \mathbb{X}_{1} \text { is } \xi_{i} \text { and } \mathbb{X}_{2} \text { is } \eta_{j} \text { then } \mathbb{Y} \text { is } g(i / k, j / k)
$$

where $i, j=1,2, \ldots, k$, respectively. Hence, we get an uncertain system $f$. From a detailed investigation using definition of uncertain system and Liu's inference rule, we know that the uncertain system is

$$
f\left(\alpha_{1}, \alpha_{2}\right)=g(i / k, j / k), \operatorname{if}\left(\alpha_{1}, \alpha_{2}\right) \in D_{i, j}
$$

Then we have

$$
\begin{aligned}
\sup _{\left(\alpha_{1}, \alpha_{2}\right) \in D}\left|f\left(\alpha_{1}, \alpha_{2}\right)-g\left(\alpha_{1}, \alpha_{2}\right)\right| & =\max _{i, j \in\{1,2, \ldots, k\}}\left\{\sup _{\left(\alpha_{1}, \alpha_{2}\right) \in D_{i, j}}\left|f\left(\alpha_{1}, \alpha_{2}\right)-g\left(\alpha_{1}, \alpha_{2}\right)\right|\right\} \\
& =\max _{i, j \in\{1,2, \ldots, k\}}\left\{\sup _{\left(\alpha_{1}, \alpha_{2}\right) \in D_{i, j}}\left|g(i / k, j / k)-g\left(\alpha_{1}, \alpha_{2}\right)\right|\right\} \\
& \leq \max _{i, j \in\{1,2, \ldots, k\}} \epsilon=\epsilon .
\end{aligned}
$$

The theorem is proved.

Theorem 1 shows that the uncertain systems can approximate continuous functions. Next, we will extend the result in the sense of mean square convergence.

Theorem 2. For any given function $g$ on a compact set $D \subset \Re^{m}$ and any given $\epsilon>0$, there exists an uncertain system $f$ such that

$$
\left(\int_{D}\left|f\left(\alpha_{1}, \alpha_{2}, \ldots, \alpha_{m}\right)-g\left(\alpha_{1}, \alpha_{2}, \ldots, \alpha_{m}\right)\right|^{2} \mathrm{~d} \alpha_{1} \mathrm{~d} \alpha_{2} \ldots \mathrm{d} \alpha_{m}\right)^{1 / 2}<\epsilon .
$$

Proof. Without loss of generality, let $g$ be a real-valued function with two variables. Note that $D$ is a compact set, and we have $\int_{D}\left|g\left(\alpha, \alpha_{2}\right)\right|^{2} \mathrm{~d} \alpha_{1} \mathrm{~d} \alpha_{2}<\infty$. Then there exists a continuous function $h$ on $D$ such that

$$
\left(\int_{D}\left|f\left(\alpha_{1}, \alpha_{2}\right)-g\left(\alpha_{1}, \alpha_{2}\right)\right|^{2} \mathrm{~d} \alpha_{1} \mathrm{~d} \alpha_{2}\right)^{1 / 2}<\epsilon / 2 .
$$

It follows from Theorem 1 that there exists an uncertain system $f$ satisfying

$$
\sup _{\left(\alpha_{1}, \alpha_{2}\right) \in D}\left|f\left(\alpha_{1}, \alpha_{2}\right)-h\left(\alpha_{1}, \alpha_{2}\right)\right|<\epsilon /\left(2 V^{1 / 2}\right)
$$


where $V=\int_{D} \mathrm{~d} \alpha_{1} \mathrm{~d} \alpha_{2}$. Hence,

$$
\begin{aligned}
\left(\int_{D}\left|f\left(\alpha_{1}, \alpha_{2}\right)-g\left(\alpha_{1}, \alpha_{2}\right)\right|^{2} \mathrm{~d} \alpha_{1} \mathrm{~d} \alpha_{2}\right)^{1 / 2} \leq & \left(\int_{D}\left|f\left(\alpha_{1}, \alpha_{2}\right)-h\left(\alpha_{1}, \alpha_{2}\right)\right|^{2} \mathrm{~d} \alpha_{1} \mathrm{~d} \alpha_{2}\right)^{1 / 2} \\
& +\left(\int_{D}\left|h\left(\alpha_{1}, \alpha_{2}\right)-g\left(\alpha_{1}, \alpha_{2}\right)\right|^{2} \mathrm{~d} \alpha_{1} \mathrm{~d} \alpha_{2}\right)^{1 / 2} \\
& <\left(\int_{D} \frac{\epsilon^{2}}{4 V} \mathrm{~d} \alpha_{1} \mathrm{~d} \alpha_{2}\right)^{1 / 2}+\epsilon / 2 \\
= & \epsilon .
\end{aligned}
$$

Theorem 2 shows that uncertain systems are capable of $L^{2}$ approximating functions which are not required continuously, including simple functions and piecewise continuous function.

\section{Conclusions}

In this paper, we prove that uncertain systems are universal approximators, and a method to construct an uncertain system to approximate any continuous function on a compact set to arbitrary accuracy is given. Hence, uncertain systems are capable of approximating any continuous function on a compact set. Furthermore, uncertain systems are capable of approximating functions, which are not continuous, in the sense of mean square convergence.

Acknowledgements

This work was supported by National Natural Science Foundation of China Grant No. 61304182.

\footnotetext{
Author details

${ }^{1}$ School of Applied Mathematics, Central University of Finance and Economics, Beijing 100081, China. ${ }^{2}$ Department of Risk Management and Insurance, Nankai University, Tianjin 300071, China.
}

Received: 17 April 2014 Accepted: 23 April 2014

Published: 7 May 2014

\section{References}

1. Zadeh, L: Fuzzy sets. Inf. Control. 8, 338-353 (1965)

2. Mamdani, EH: Applications of fuzzy algorithms for control of a simple dynamic plant. Proc. IEEE. 121(12), 1585-1588 (1974)

3. Takagi, T, Sugeno, M: Fuzzy identification of system and its applications to modeling and control. IEEE Trans. Syst. Man Cybernatics. 15(1), 116-132 (1985)

4. Lukasiewicz, J: Selected Works - Studies in Logic and the Foundations of Mathematics. North-Holland, Amsterdam, Warsaw (1970)

5. Mamdani, EH: Application of fuzzy logic to approximate reasoning using linguistic synthesis. IEEE Trans. Comput. C-26(12), 1182-1191 (1977)

6. Wang, L: Fuzzy systems are universal approximators. In: IEEE International Conference on Fuzzy Systems, pp. 1163-1170, San Diego, 8-12 March 1992

7. Liu, B: Uncertain set theory and uncertain inference rule with application to uncertain control. J. Uncertain Syst. 4(2), 83-98 (2010)

8. Gao, X, Gao, Y, Ralescu, DA: On Liu's inference rule for uncertain systems. Int. J. Uncertainty Fuzziness Knowledge-Based Syst. 18(1), 1-11 (2010)

9. Gao, Y: Uncertain inference control for balancing an inverted pendulum. Fuzzy Optimization Decis. Making. 11(4), 481-492 (2012)

10. Liu, B: Uncertain logic for modeling human language. J. Uncertain Syst. 5(1), 3-20 (2011)

11. Liu, B: Uncertainty Theory. 2nd ed. Springer, Berlin (2007)

12. Gao, X: Some properties of continuous uncertain measure. Int. J. Uncertainty Fuzziness Knowledge-Based Syst. 17(3), 419-426 (2009)

13. Liu, B: Theory and Practice of Uncertain Programming. 2nd ed. Springer, Berlin (2009)

14. Liu, B: Fuzzy process, hybrid process and uncertain process. J. Uncertain Syst. 2(1), 3-16 (2008)

15. Li, X, Liu, B: Hybrid logic and uncertain logic. J. Uncertain Syst. 3(2), 83-94 (2009) 
16. Liu, B: Uncertain entailment and modus ponens in the framework of uncertain logic. J. Uncertain Syst. 3(4), 243-251 (2009)

17. Liu, B: Uncertainty Theory: a Branch of Mathematics for Modeling Human Uncertainty. Springer, Berlin (2010)

18. Liu, B: Some research problems in uncertainty theory. J. Uncertain Syst. 3(1), 3-10 (2009)

19. Liu, B: Membership functions and operational law of uncertain sets. Fuzzy Optimization Decis. Making. 11(4), 387-410 (2012)

20. Liu, B: A new definition of independence of uncertain sets. Fuzzy Optimization Decis. Making. 12(4), 451-461 (2013)

doi:10.1186/2195-5468-2-13

Cite this article as: Peng and Chen: Uncertain systems are universal approximators. Journal of Uncertainty Analysis and Applications 2014 2:13.

Submit your manuscript to a SpringerOpen ${ }^{\circ}$ journal and benefit from:

$\checkmark$ Convenient online submission

- Rigorous peer review

- Immediate publication on acceptance

- Open access: articles freely available online

- High visibility within the field

- Retaining the copyright to your article

Submit your next manuscript at $\boldsymbol{\triangleright}$ springeropen.com 